\title{
RIGIDITY OF WEIGHTED COMPOSITION OPERATORS ON $\boldsymbol{H}^{p}$
}

\section{Mikael Lindström, Santeri Miihkinen and Pekka J. Nieminen}

\author{
Åbo Akademi University, Department of Mathematics \\ FI-20500 Åbo, Finland; mikael.lindstrom@abo.fi \\ Åbo Akademi University, Department of Mathematics \\ FI-20500 Åbo, Finland; santeri.miihkinen@abo.fi \\ University of Turku, Department of Mathematics and Statistics \\ FI-20014 University of Turku, Finland; pekka.nieminen@utu.fi
}

\begin{abstract}
We show that every non-compact weighted composition operator $f \mapsto u \cdot(f \circ \phi)$ acting on a Hardy space $H^{p}$ for $1 \leq p<\infty$ fixes an isomorphic copy of the sequence space $\ell^{p}$ and therefore fails to be strictly singular. We also characterize those weighted composition operators on $H^{p}$ which fix a copy of the Hilbert space $\ell^{2}$. These results extend earlier ones obtained for unweighted composition operators.
\end{abstract}

\section{Introduction and main results}

Let $\mathbf{D}$ be the open unit disc in the complex plane $\mathbf{C}$ and fix analytic maps $u: \mathbf{D} \rightarrow \mathbf{C}$ and $\phi: \mathbf{D} \rightarrow \mathbf{D}$. The weighted composition operator $u C_{\phi}$ is defined by

$$
\left(u C_{\phi}\right) f=u \cdot(f \circ \phi)
$$

for $f: \mathbf{D} \rightarrow \mathbf{C}$ analytic. Boundedness and compactness properties of such operators acting on the classical Hardy spaces $H^{p}$ were characterized in terms of Carleson measures in $[1,2]$ (see also [3]). An obvious necessary condition for the boundedness of $u C_{\phi}: H^{p} \rightarrow H^{q}$ is that $u=\left(u C_{\phi}\right) 1 \in H^{q}$.

The purpose of this work is to study the qualitative properties of non-compact weighted composition operators on the Hardy spaces $H^{p}$, extending the results obtained in [6] for unweighted composition operators. It turns out that the weighted composition operators exhibit the exact same rigidity phenomena as the unweighted ones. We also refer the reader to the recent parallel work [7] in the context of Volterra-type integral operators, where some of our ideas originate from.

Recall that if $X$ is a Banach space and $T: X \rightarrow X$ is a linear operator, then $T$ is called strictly singular if the restriction of $T$ to any infinite-dimensional subspace of $X$ is not an isomorphism (equivalently, it is not bounded below). For a detailed discussion of strict singularity and related notions in the context of Hardy spaces and composition operators, see [6] and the references therein.

Our first result is a generalization of [6, Thm 1.2] and shows, in particular, that the notions of compactness and strict singularity coincide for weighted composition operators on $H^{p}$. Here we employ the usual test functions

$$
g_{a}(z)=\frac{\left(1-|a|^{2}\right)^{1 / p}}{(1-\bar{a} z)^{2 / p}}, \quad z \in \mathbf{D},
$$

where $a \in \mathbf{D}$. They always satisfy $\left\|g_{a}\right\|_{H^{p}}=1$.

https://doi.org/10.5186/aasfm.2020.4537

2010 Mathematics Subject Classification: Primary 47B33, 47B10.

Key words: Hardy space, strict singularity, weighted composition operator. 
Theorem 1. Let $1 \leq p<\infty$ and suppose that $u C_{\phi}$ is bounded and non-compact $H^{p} \rightarrow H^{p}$. Then $u C_{\phi}$ fixes an isomorphic copy of $\ell^{p}$ in $H^{p}$. More precisely, there exists a sequence $\left(a_{n}\right)$ in $\mathbf{D}$ such that $\left(g_{a_{n}}\right)$ is equivalent to the natural basis of $\ell^{p}$ and $u C_{\phi}$ is bounded below on the closed linear span of $\left(g_{a_{n}}\right)$.

We next determine under which conditions a weighted composition operator on $H^{p}$ with $p \neq 2$ fixes a copy of the Hilbert space $\ell^{2}$. In the unweighted case (see $[6$, Thm 1.4]) this is the case precisely when the boundary contact set

$$
E_{\phi}=\{\zeta \in \mathbf{T}:|\phi(\zeta)|=1\}
$$

has positive measure. It turns out that this result holds in the weighted case as well. The first half is established in the following theorem, where we also allow for the possibility that the target space of the operator is a larger Hardy space than the domain.

Theorem 2. Let $1 \leq q \leq p<\infty$ and suppose that $u C_{\phi}$ is bounded $H^{p} \rightarrow H^{q}$. If $u \neq 0$ and $m\left(E_{\phi}\right)>0$, then $u C_{\phi}$ fixes an isomorphic copy of $\ell^{2}$ in $H^{p}$. Here $m$ is the normalised Lebesgue measure on $\mathbf{T}$.

In the converse direction we have the following result.

Theorem 3. Let $1 \leq p<\infty$ and suppose that $u C_{\phi}$ is bounded $H^{p} \rightarrow H^{p}$ with $m\left(E_{\phi}\right)=0$. If $u C_{\phi}$ is bounded below on an infinite-dimensional subspace $M \subset H^{p}$, then $M$ contains an isomorphic copy of $\ell^{p}$. In particular, if $p \neq 2$, then $u C_{\phi}$ does not fix any isomorphic copies of $\ell^{2}$ in $H^{p}$.

The last statement of the theorem is due to the fact that $\ell^{p}$ and $\ell^{2}$ are totally incomparable spaces for $p \neq 2$.

\section{Proofs}

Towards the proof of Theorem 1 we first state the following lemma.

Lemma 4. Let $u \in H^{p}$ and $\phi: \mathbf{D} \rightarrow \mathbf{D}$ be analytic. For $\epsilon>0$, define

$$
F_{\epsilon}=\{\zeta \in \mathbf{T}:|\phi(\zeta)-1|<\epsilon\} .
$$

Then

$$
\begin{gathered}
\lim _{a \rightarrow 1} \int_{\mathbf{T} \backslash F_{\epsilon}}\left|\left(u C_{\phi}\right) g_{a}\right|^{p} d m=0 \quad \text { for each } \epsilon>0, \\
\lim _{\epsilon \rightarrow 0} \int_{F_{\epsilon}}\left|\left(u C_{\phi}\right) g_{a}\right|^{p} d m=0 \quad \text { for each } a \in \mathbf{D} .
\end{gathered}
$$

Proof. Let $\epsilon>0$ be fixed and consider $\zeta \in \mathbf{T} \backslash F_{\epsilon}$ for which the radial limit $\phi(\zeta)$ exists. Then, if $|a-1|<\epsilon / 2$, we have

$$
|1-\bar{a} \phi(\zeta)| \geq|1-\phi(\zeta)|-|1-a|>\epsilon / 2
$$

and so

$$
\int_{\mathbf{T} \backslash F_{\epsilon}}\left|\left(u C_{\phi}\right) g_{a}\right|^{p} d m \leq\left(1-|a|^{2}\right) \int_{\mathbf{T} \backslash F_{\epsilon}} \frac{|u|^{p}}{|1-\bar{a} \phi|^{2}} d m \leq \frac{4\left(1-|a|^{2}\right)}{\epsilon^{2}}\|u\|_{H^{p}}^{p} .
$$

Since this tends to 0 as $a \rightarrow 1$, we obtain the first part of the lemma.

The second part follows from the absolute continuity of the measure $F \mapsto$ $\int_{F}\left|\left(u C_{\phi}\right) g_{a}\right|^{p} d m$ and the fact that $m\left(F_{\epsilon}\right) \rightarrow m(\{\zeta \in \mathbf{T}: \phi(\zeta)=1\})=0$ as $\epsilon \rightarrow 0$. Note that $g_{a} \in H^{\infty}$ and hence $\left(u C_{\phi}\right) g_{a} \in L^{p}(\mathbf{T}, m)$. 
Proof of Theorem 1. Since $u C_{\phi}$ is non-compact, we may find a sequence $\left(a_{n}\right)$ in $\mathbf{D}$ such that $\left|a_{n}\right| \rightarrow 1$ and $\left\|\left(u C_{\phi}\right) g_{a_{n}}\right\|_{H^{p}} \geq c>0$ for all $n$. This is a consequence of the compactness characterization of $u C_{\phi}$ in terms of vanishing Carleson measures; see [1, Theorem 3.5]. By passing to a convergent subsequence of $\left(a_{n}\right)$ and utilizing a suitable rotation, we may assume that $a_{n} \rightarrow 1$.

We now proceed exactly as in the unweighted case (see the proof of Theorem 1.2 in [6] for the details of the following argument). First, by invoking Lemma 4 repeatedly, we may extract a subsequence of $\left(a_{n}\right)$, still denoted by $\left(a_{n}\right)$, such that the image sequence $\left(\left(u C_{\phi}\right) g_{a_{n}}\right)$ in $H^{p}$ is equivalent to the standard basis of $\ell^{p}$, that is,

$$
\left\|\sum_{n=1}^{\infty} \alpha_{n}\left(u C_{\phi}\right) g_{a_{n}}\right\|_{H^{p}} \sim\left\|\left(\alpha_{n}\right)\right\|_{p} \quad \text { for }\left(\alpha_{n}\right) \in \ell^{p}
$$

where $A \sim B$ for quantities $A$ and $B$ means that $c A \leq B \leq C A$ for some positive numbers $c$ and $C$.

Then a second application of Lemma 4 to the functions $g_{a_{n}}$ (taking $u=1$ and $\phi(z)=z$ ) produces a further subsequence of $\left(a_{n}\right)$, which we continue to denote by $\left(a_{n}\right)$, such that also

$$
\left\|\sum_{n=1}^{\infty} \alpha_{n} g_{a_{n}}\right\|_{H^{p}} \sim\left\|\left(\alpha_{n}\right)\right\|_{p} \quad \text { for }\left(\alpha_{n}\right) \in \ell^{p} .
$$

By combining the preceding two norm estimates we see that $u C_{\phi}$ restricts to a linear isomorphism on the closed linear span of $\left(g_{a_{n}}\right)$.

Proof of Theorem 2. Since $m\left(E_{\phi}\right)>0,[6$, Prop. 3.2] shows that there exists a sequence of integers $\left(n_{k}\right)$ satisfying $\inf _{k}\left(n_{k+1} / n_{k}\right)>1$ and a constant $c_{1}>0$ such that $\left\|\sum_{k} \alpha_{k} \phi^{n_{k}}\right\|_{H^{1}} \geq c_{1}\left\|\left(\alpha_{k}\right)\right\|_{2}$ for all $\left(\alpha_{k}\right) \in \ell^{2}$. Our goal is to prove a weighted version of this estimate, that is, for some constant $c>0$,

$$
\left\|u \sum_{k} \alpha_{k} \phi^{n_{k}}\right\|_{H^{1}} \geq c\left\|\left(\alpha_{k}\right)\right\|_{2} \text {. }
$$

Since Paley's theorem (see e.g. [4, p. 104]) implies that the closed linear span $M=$ $\left[z^{n_{k}}: k \geq 1\right]$ in $H^{p}$ is isomorphic to $\ell^{2}$, inequality $(1)$ implies that $u C_{\phi}$ is an isomorphism from $M$ into $H^{1}$. This yields the theorem because $\|f\|_{H^{q}} \geq\|f\|_{H^{1}}$ for all $f \in H^{q}$.

To establish (1), we first note that since $u \neq 0$, we have $|u|>0$ a.e. on $\mathbf{T}$. Thus, for a given $\epsilon>0$ there exists a set $F \subset \mathbf{T}$ with $m(\mathbf{T} \backslash F)<\epsilon$ such that $|u|>c_{2}$ on $F$ for some constant $c_{2}=c_{2}(\epsilon)>0$. Then, using Hölder's inequality and the boundedness of $C_{\phi}$ on $H^{2}$, we get

$$
\int_{\mathbf{T} \backslash F}\left|\sum_{k} \alpha_{k} \phi^{n_{k}}\right| d m \leq \sqrt{m(\mathbf{T} \backslash F)}\left\|\sum_{k} \alpha_{k} \phi^{n_{k}}\right\|\left\|_{2} \leq \sqrt{\epsilon} \cdot c_{3}\right\|\left(\alpha_{k}\right) \|_{2}
$$

for some constant $c_{3}>0$. On combining these estimates we obtain

$$
\left\|u \sum_{k} \alpha_{k} \phi^{n_{k}}\right\|_{H^{1}} \geq c_{2} \int_{F}\left|\sum_{k} \alpha_{k} \phi^{n_{k}}\right| d m \geq c_{2}\left(c_{1}-\sqrt{\epsilon} \cdot c_{3}\right)\left\|\left(\alpha_{k}\right)\right\|_{2} .
$$

In particular, choosing $\epsilon=\left(c_{1} / 2 c_{3}\right)^{2}$ here proves (1) with $c=\frac{1}{2} c_{2} c_{1}$. This completes the proof of the theorem. 
Proof of Theorem 3. Since $M$ is infinite-dimensional, there exists a sequence $\left(f_{n}\right)$ in $M$ such that $\left\|f_{n}\right\|_{H^{p}}=1$ and $f_{n} \rightarrow 0$ uniformly on compact subsets of $\mathbf{D}$; for instance, we can choose $f_{n}(0)=f_{n}^{\prime}(0)=\cdots=f_{n}^{(n)}(0)=0$ for all $n$.

For each $k \geq 1$, define $E_{k}=\left\{\zeta \in \mathbf{T}:|\phi(\zeta)|>1-\frac{1}{k}\right\}$. We have $m\left(E_{k}\right) \rightarrow$ $m\left(E_{\phi}\right)=0$ as $k \rightarrow \infty$ and therefore

$$
\lim _{k \rightarrow \infty} \int_{E_{k}}\left|\left(u C_{\phi}\right) f_{n}\right|^{p} d m=0 .
$$

On the other hand, since $f_{n} \circ \phi$ converges to zero uniformly on $\mathbf{T} \backslash E_{k}$ as $n \rightarrow \infty$ and $u \in H^{p}$,

$$
\lim _{n \rightarrow \infty} \int_{\mathbf{T} \backslash E_{k}}\left|\left(u C_{\phi}\right) f_{n}\right|^{p} d m=0 .
$$

Since $u C_{\phi}$ is bounded below on $M$, we also have $\left\|\left(u C_{\phi}\right) f_{n}\right\|_{H^{p}} \geq c$ for all $n$ and some constant $c>0$. Using a gliding hump argument based on a repeated application of (2) and (3) (akin to the proof of [6, Prop. 3.3] in the unweighted case), we may extract a subsequence $\left(f_{n_{j}}\right)$ such that the sequence $\left(\left(u C_{\phi}\right) f_{n_{j}}\right)$ is equivalent to the standard basis of $\ell^{p}$. Since $u C_{\phi}$ is bounded below on the closed linear span $\left[f_{n_{j}}: j \geq 1\right] \subset M$, we conclude that $u C_{\phi}$ fixes a copy of $\ell^{p}$ in $M$.

To conclude, we record a couple of questions on the space $B M O A$, which consists of those functions in $H^{2}$ that have boundary values of bounded mean oscillation.

Q1. Does every bounded and non-compact $u C_{\phi}: B M O A \rightarrow B M O A$ fix an isomorphic copy of $c_{0}$ (the space of null sequences)? This is known to be true for (unweighted) composition operators; see [5, Section 3] and the discussion in [6, p. 625].

Q2. Do Theorems 2 and 3 have versions for $u C_{\phi}$ on $B M O A$ ? More specifically, is it true that $u C_{\phi}: B M O A \rightarrow B M O A$ fixes an isomorphic copy of $\ell^{2}$ if and only if $u \neq 0$ and $m\left(E_{\phi}\right)>0$ ? In the unweighted case, a partial result of this kind is obtained in [6, Prop. 4.1].

Acknowledgements. The first and the second authors were partially supported by the Academy of Finland project 296718.

\section{References}

[1] Contreras, M. D., and A. G. Hernández-Díaz: Weighted composition operators on Hardy spaces. - J. Math. Anal. Appl. 263:1, 2001, 224-233.

[2] Contreras, M. D., and A. G. Hernández-Díaz: Weighted composition operators between different Hardy spaces. - Integral Equations Operator Theory 46, 2003, 165-188.

[3] C̆UC̆Ković, Z., and R. ZhaO: Weighted composition operators between different weighted Bergman spaces and different Hardy spaces. - Illinois J. Math. 51:2, 2007, 479-498.

[4] Duren, P. L.: Theory of $H^{p}$ spaces. - Academic Press, 1970; reprinted by Dover, 2000.

[5] Laitila, J., P. J. Nieminen, E. Saksman, and H.-O. Tylli: Compact and weakly compact composition operators on BMOA. - Complex Anal. Oper. Theory 7:1, 2013, 163-181.

[6] Laitila, J., P. J. Nieminen, E. Saksman, and H.-O. Tylli: Rigidity of composition operators on the Hardy space $H^{p}$. - Adv. Math. 319, 2017, 610-629.

[7] Mirhkinen, S., P. J. Nieminen, E. Saksman, and H.-O. Tylli: Structural rigidity of generalised Volterra operators on $H^{p}$. - Bull. Sci. Math. 148, 2018, 1-13. 